УДК 621.372 .22

\title{
ПРЯМОУГОЛЬНАЯ ДИЭЛЕКТРИЧЕСКАЯ РЕЗОНАНСНАЯ АНТЕННА В ФОРМЕ КОВРА СЕРПИНСКОГО С ИСПОЛЬЗОВАНИЕМ ТЕФЛОНА ДЛЯ Х-ДИАПАЗОНА
}

\author{
ДИПАЛИ СОРЕН ${ }^{1}$, РОУДРА ГХАТАК $^{2}$, Р. К. МИШРА ${ }^{3}$, Д. Р. ПОДДАР ${ }^{4}$ \\ ${ }^{1}$ Технический университет Чхаттисгарх Свами Вивекананд, \\ Индия, Бхилаи, Чхаттисгарх \\ ${ }^{2}$ Нацииональный технологический институт Дургапур, \\ Индия, Дургапур, Западная Бенгалия \\ ${ }^{3}$ Берхампурский университет, \\ Индия, Берхампур, Орисса \\ ${ }^{4}$ Джадавпурский университет, \\ Индия, Калькутта, Западная Бенгалия
}

\begin{abstract}
Аннотация. В статье представлена широкополосная прямоугольная диэлектрическая резонансная антенна DRA (dielectric resonator antenna) в форме ковра Серпинского, работающая в Х-диапазоне, которая характеризуется моделью диэлектрического волновода DWM (dielectric waveguide model). Для снижения стоимости DRA в качестве материала использован дешевый тефлон. Результаты моделирования проверены с помощью реализованного прототипа. В статье обычная прямоугольная DRA сравнивается с прямоугольными DRA в форме фрактала первой и второй итераций. Обсуждается методология проектирования антенны и ее резонансные и радиационные характеристики. Обоснованность полученных результатов подтверждается близким совпадением экспериментальных и моделированных результатов. Измерения на прототипе показали ширину полосы пропускания $48 \%$, определяемую по полному входному сопротивлению. При этом она охватывает всю полосу частот X- с подобной диаграммой направленности во всей полосе и усилением 7,5 дБи в диапазоне 9,0-11,5 ГГц.
\end{abstract}

Ключевые слова: широкополосный; диэлектрическая резонаторная антенна; фрактал; ковер Серпинского

\section{1. ВВЕДЕНИЕ}

Существующим и будущим системам беспроводной связи нужны антенны, способные поддерживать все более высокие скорости передачи данных и повышенную плотность пользователей. Это вызывает повышенный интерес к разработке широкополосных и многоканальных антенн, в том числе в виде нескольких антенн, обычно с одинаковыми характеристиками, которые физически отделены друг от друга. Это выгодно для индустрии мобильной связи, поскольку позволяет нескольким поль- зователям совместно использовать ограниченный спектр и избегать помех в совмещенном канале.

Из-за своего небольшого размера в устройствах мобильной связи в качестве излучателей обычно используют плоские микрополосковые антенны. В случае микрополосковой антенны, омические и поверхностные волновые потери возрастают с ростом частоты. С другой стороны, диэлектрическая резонансная антенна DRA (dielectric resonator antenna), предложенная в 1983 году [1], имеет несколько преимуществ, таких как низкие омические по- 


\section{REFERENCES}

1. Long, S. A.; Mcallister, M. W.; Shen, L. C. "The resonant cylindrical dielectric cavity antenna," IEEE Trans. Antennas Propag., Vol. 31, No. 3, p. 406-412, 1983. DOI: 10.1109/TAP.1983.1143080.

2. Luk, K. M.; Leung, K. W. (eds.). Dielectric Resonator Antennas. England: Research Studies Press Ltd. 2002.

3. Denidni, T. A.; Coulibaly, Y.; Boutayab, H. "Hybrid dielectric resonator antenna with circular mushroom-like structure for gain improvement," IEEE Trans. Antennas Propag., Vol. 57. No. 4, p. 1043-1049, 2009. DOI: 10.1109/TAP.2009.2015809.

4. Kishk, A. A.; Yin, Y.; Glisson, A. W. "Conical dielectric resonator antennas for wide-band applications," IEEE Trans.Antennas Propag., Vol. 50, No. 4, p. 469-474, Apr. 2002. DOI: $10.1109 /$ TAP.2002.1003382.

5. Kishk, A. A. "Tetrahedron and triangular dielectric resonator antenna with wideband performance," Proc. of IEEE AP-S Int. Symp., 16-21 June 2002, San Antonio, USA. IEEE, 2002, Vol. 4, p. 462-465. DOI: 10.1109/APS.2002.1017021.

6. Kishk, A. A. "Wide-band truncated tetrahedron dielectric resonator antenna excited by a coaxial probe," IEEE Trans. Antennas Propag., Vol. 51, No. 10, p. 2913-2917, Oct. 2003. DOI: $10.1109 /$ TAP.2003.816300.

7. Denidni, Tayeb A.; Rao, Q.; Sebak, A. R. "Broadband L-shaped dielectric resonator antenna," IEEE Antennas Wireless Propag. Lett., Vol. 4, p. 453-454, 2005. DOI: 10.1109/LAWP.2005.860198.

8. Chang, T.-H.; Huang, Y.-C.; Su, W.-F.; Kiang, J.-F. "Wideband dielectric resonator antenna with a tunnel," IEEE Antennas Wireless Propag. Lett., Vol. 7, p. 275-278, 2008. DOI: 10.1109/LAWP.2008.928477.

9. Coulibaly,Y; Denidni, Tayeb A.; Boutayeb, Halim. "Broadband microstrip-fed dielectric resonator antenna for X-band applications," IEEE Antennas Wireless Propag. Lett., Vol. 7, p. 341-3445, 2008. DOI: 10.1109/LAWP.2008.921326.
10. Lapierre, M.; Antar, Y. M. M.; Ittipiboon, A.; Petosa, A. "A wideband monopole antenna using dielectric resonator loading," IEEE Antennas Propagation Society Int. Symp. Dig., 22-27 June 2003, Columbus, USA. IEEE, 2003, Vol. 3, p. 16-19. DOI: 10.1109/APS.2003.1219778.

11. Junker, G. P.; Kishk, A. A.; Glisson, A. W.; Kajifez, D. "Effect of air gap on cylindrical dielectric resonator antenna operating in $\mathrm{TM}_{01}$ mode," Electronics Lett., Vol. 30, No. 2, p. 97-98, Jan. 20, 1994. DOI: 10.1049/el: 19940114.

12. Junker, G. P.; Kishk, A. A.; Glisson, A. W.; Kajfez, D. "Effect of an air gap around the coaxial probe exciting a cylindrical dielectric resonator antenna," Electronics Lett., Vol. 30, No. 3, p. 177-178, 1994. DOI: 10.1049/el:19940191.

13. Coulibaly, Y.; Denidni, T. A.; Talbi, L. "Design of a broadband hybrid dielectric resonator antenna for X-band applications," J. Electromagn. Waves Appl., Vol. 20, No. 12, p. 1629-1642, 2006. DOI: 10.1163/156939306779292354.

14. Rezaei, P.; Hakkak, M.; Forooraghi, K. "Effect of magnetic layer on the microstrip-excited rectangular dielectric resonator antennas bandwidth," J. Electromagn. Waves Appl., Vol. 21, No. 7, p. 915-927, 2007. DOI: 10.1163/156939307780749011.

15. Kingsley, S. P.; O’Keefe, S. G. "Beam steering and monopulse processing of probe-fed dielectric resonator antennas," IEE Proc. - Radar, Sonar Navigation, Vol. 146, No. 3 , p. 121-125, June 1999. DOI: 10.1049/ip-rsn:19990307.

16. Ghatak, R.; Poddar, D. R.; Mishra, R. K. "Design of Sierpinski gasket fractal microstrip antenna using real coded genetic algorithm," IET Microwave Antennas Propag., Vol. 3, No. 7, p. 1133-1140, 2009. DOI: 10.1049/iet-map.2008.0257.

17. Soren, Dipali; Ghatak, Rowdra; Mishra, R. K.; Poddar, D. R. "Sierpinski carpet fractal pattern embedded multisegment rectangular dielectric resonator antenna for Wi-MAX and WLAN application," Proc. of Int. Symp. on Microwaves and Millimeterwaves: Basics and Technology, ISoMM 09, 14-16 Jan. 2009, Kolkata, India. 2009. 
18. Karmakar, D. P.; Soren, Dipali; Ghatak, Rowdra; Poddar, Dipak R.; Mishra, Rabindra K. "A wideband Sierpinski carpet fractal cylindrical dielectric resonator antenna for X-band application," Proc. of IEEE Applied Electromagnetics Conf., 14-16 Dec. 2009, Kolkata, India. IEEE, 2009. DOI: 10.1109/AEMC.2009.5430713.

19. Gangwar, R. K.; Singh, S. P.; Kumar, D. "A modified fractal rectangular curve dielectric resonator antenna for WiMAX application," PIER C, Vol. 12, p. 37-51, 2010. DOI: 10.2528/PIERC09111303.

20. Guha, D.; Banerjee, A.; Kumar, C.; Antar, Y. M. M. "Higher order mode excitation for high-gain broadside radiation from cylindrical dielectric resonator antennas," IEEE Trans. Antennas Propag., Vol. 60, No. 1, p. 71-77, Jan. 2012. DOI: 10.1109/TAP.2011.2167922.

21. Motevasselian, A.; Ellgardt, A.; Jonsson, B. L. G. "A circularly polarized cylindrical dielectric resonator antenna using a helical exciter," IEEE Trans. Antennas Propag., Vol. 61, No. 3, p. 1439-1443, Mar. 2013. DOI: $10.1109 /$ TAP.2012.2229954

22. Sun, Y. X.; Leung, K. W. "Dual-band and wideband dual-polarized cylindrical dielectric resonator antennas," IEEE Antennas Wireless Propag. Lett., Vol. 12, p. 384-387, Mar. 2013. DOI: 10.1109/LAWP.2013.2251993.

23. Fang, X. S.; Leung, K. W.; Luk, K. M. "Theory and experiment of three-port polarization-diversity cylindrical dielectric resonator antenna," IEEE Trans. Antennas Propag., Vol. 62, No. 10 , p. 4945-4951, Oct. 2014. DOI: 10.1109/TAP.2014.2341698.

24. Pan, Y. M.; Zheng, S. Y.; Hu, B. J. "Design of dual-band omnidirectional cylindrical dielectric resonator antenna," IEEE Antennas Wireless Propag. Lett., Vol. 13, p. 710-713, Apr. 2014. DOI: 10.1109/LAWP.2014.2314745.

25. Avadanei, O. G.; Banciu, M. G.; Nedelcu, L. "Higher-order modes in high-permittivity cylindrical dielectric resonat- or antenna excited by an off-centered rectangular slot," IEEE Antennas Wireless Propag. Lett., Vol. 13, p. 1585-1588, Aug. 2014. DOI: $10.1109 /$ LAWP.2014.2344860.

$26=24$. Pan, Y. M.: Zheng. S. Y.: Hu, B. J. "Design of dual-band omnidirectional cylindrical dielectric resonator antenna," IEEE Antennas and Wireless Propag. Lett. Vol. 13, p. 710-713, Apr. 2014. DOI: 10.1109/LAWP.2014.2314745.

27. Pan, Y. M.; Zheng, S. Y.; Li, W. "Dual-band and dual-sense omnidirectional circularly polarized antenna," IEEE Antennas Wireless Propag. Lett., Vol. 13, p. 706-709, Apr. 2014. DOI: 10.1109/LAWP.2014.2314744.

28. Khalily, M.; Kamarudin, M. R.; Mokayef, M. Jamaluddin, M. H. "Omnidirectional circularly polarized dielectric resonator antenna for 5.2-GHz WLAN applications." IEEE Antennas Wireless Propag. Lett., Vol. 13, p. 443-446, Mar. 2014. DOI: 10.1109/LAWP.2014.2309657.

29. Altaf, Amir; Yang, Youngoo; Lee, Kang-Yoon; Hwang, Keum Cheol. "Circularly polarized Spidron fractal dielectric resonator antenna," IEEE Antennas Wireless Propag. Lett., Vol. 14. p. 1806-1809, April, 2015. DOI: 10.1109/LAWP.2015.2427373.

30. Maity, Sudipta; Gupta, Bhaskar. "Experimental investigations on wideband triangular dielectric resonator antenna," IEEE Trans. Antennas Propag., Vol. 64, No. 12, p. 5483-5486, Dec 2016. DOI: 10.1109/TAP.2016.2607765.

31. Tang, Hui; Chen, Jian-Xin; Yang, Wen-Wen; Zhou, Li-Heng; Li, Wenhua. "Differential dual-band dual-polarized dielectric resonator antenna," IEEE Trans. Antennas Propag., Vol. 65 , No. 2, p. $855-860$, Feb 2017. DOI: 10.1109/TAP.2016.2630661. 